\title{
DETERMINAÇÃO DA ENERGIA DE ATIVAÇÃO NA PRODUÇÃO DE ACETATO DE EUGENILA VIA CATÁLISE HETEROGÊNEA
}

\author{
R.A. $\operatorname{LOSS}^{1}$, D.A.LAROQUE ${ }^{1}$, M.J.A.SILVA ${ }^{1}$, J.V. OLIVEIRA ${ }^{1,}$ D. OLIVEIRA ${ }^{1,}$ H. HENSE ${ }^{1}$ \\ ${ }^{1}$ Universidade Federal de Santa Catarina, Departamento de Engenharia Química e Engenharia de \\ Alimentos \\ E-mail para contato: raquelaloss@gmail.com
}

\begin{abstract}
RESUMO - Devido à crescente busca por processos ecologicamente corretos, a catálise heterogênea tem recebido destaque por sua eficiência para alcançar transformações limpas, além de vantagens relacionadas a atividade e seletividade das reações. O presente trabalho tem como objetivo determinar a energia de ativação na produção de acetato de eugenila, empregando como catalisador a peneira molecular $4 \AA$. Para tanto, utilizou-se a equação de Arrhenius, sendo que as temperaturas avaliadas foram 40, 50, 55 e $60{ }^{\circ} \mathrm{C}$, a proporção de catalisador foi de $12 \%(\mathrm{~m} / \mathrm{m}$ de substratos) e a razão molar de eugenol e anidrido acético de 1:3. Resultados das cinéticas destrutivas mostram que as conversões em acetato de eugenila aumentaram com a elevação da temperatura. Além disso, um catalisador tem função de diminuir a energia necessária para ocorrência da reação, neste caso, a peneira molecular mostrou-se um catalisador interessante, uma vez que apresentou baixa energia de ativação quando comparada com outros catalisadores empregados em reações de esterificação. Desta forma, conclui-se que temperaturas mais elevadas contribuem para a obtenção de maiores conversões em éster de acetato de eugenila, sendo que não foi observado necessidade de temperaturas acima de $50{ }^{\circ} \mathrm{C}$ uma vez que conversões em éster próximas a $100 \%$ foram obtidas nesta condição.
\end{abstract}

\section{INTRODUÇÃO}

Durante os últimos anos, os processos, que se enquadram no conceito de química verde vem ganhando destaque no mercado de aromas e fragrâncias, representando uma meta desafiadora para a pesquisa acadêmica e industrial. Aspectos ambientais e econômicos tem despertado crescente interesse, tanto da comunidade acadêmica quanto industrial em pesquisas buscando a elaboração de processos que sejam ambientalmente favoráveis, seletivos, apresentem elevados rendimentos e fácil manipulação (Sartori et al., 2004). Dentre esses fatores, se destaca a seleção do catalisador que deve ser realizada de acordo com a reação envolvida e o produto requerido, estes devem apresentar como uma das principais características baixa energia de ativação (Dumesic; Huber; Boudart, 2008).

A catálise heterogênea tem conquistado um espaço cada vez maior na obtenção de compostos químicos para as mais variadas aplicações. Este processo tem se mostrado útil no 
desenvolvimento de processos seletivos e com geração reduzida de resíduos, tornando-as atraentes tanto do ponto de vista ambiental quanto econômico (Wender et al., 1997).

Sólidos inorgânicos porosos, como as peneiras moleculares tem grande utilidade como catalisadores e adsorventes para muitas aplicações industriais. A presença de porosidade permite que as moléculas tenham acesso a elevadas áreas superficiais, aos quais estão associadas altas atividades catalíticas e adsortivas (Beck, 1991; Kresge et al., 1992).

Os óleos essenciais são produtos de origem natural que representam uma importante vertente econômica, sendo empregados nas indústrias de perfumes, cosméticos (aromatizantes), agricultura (fungicida em plantações de banana), medicina (tratamento de doenças como asma e diabetes), nutrição (como aditivos de sabor e aroma) e alimentos (agente antimicrobiano e antioxidante) (Costa, 1994). Os alcoóis terpênicos são os constituintes presentes em maior concentração nos óleos essenciais e são responsáveis pelo aroma, além de serem ótimos substratos para a síntese de compostos de interesse nas indústrias química, farmacêutica e na conservação de alimentos (Kollmannsberger; Nitz, 1993). Entre eles destacam-se alcoóis mono e sesquiterpênicos tais como geraniol, linalol, citronelol, elemol, eugenol, mentol, entre outros.

Desta forma, o presente estudo teve como objetivo avaliar a energia de ativação na síntese de acetato de eugenila via catálise heterogênea empregando peneira molecular $4 \AA$ como catalisador da reação de acetilação do óleo essencial de folha de cravo-da-índia e anidrido acético.

\section{MATERIAL E MÉTODOS}

\subsection{Materiais}

Óleo essencial de folha de cravo-da-índia (Eugenia caryophyllus) adquirido da Ferquima Indústria e Comércio de Óleos Essenciais (São Paulo, Brasil), e anidrido acético (Vetec, 97\%) foram usados como substratos nas reações de acetilação e como catalisador foi empregado a peneira molecular $4 \AA$ (Merck Milipore).

\subsection{Efeito da temperatura na velocidade de produção de acetato de eugenila}

O efeito da temperatura na velocidade da reação foi avaliado através da Equação de Arrhenius, conforme Equação 1.

$$
\ln V=\ln V_{0}-\left(\frac{E_{a}}{R}\right)\left(\frac{1}{T}\right)
$$

Onde: V: constante cinética da reação $\left(\mathrm{h}^{-1}\right)$; $\ln$ Vo: fator pré-exponencial $\left(\mathrm{h}^{-1}\right)$; Ea: energia de ativação $\left(\mathrm{J} \mathrm{mol}^{-1}\right)$; R: constante universal dos gases $\left(8,314 \mathrm{Jmol}^{-1} \mathrm{~K}^{-1}\right)$; T: temperatura $(\mathrm{K})$. 


\section{9 a 22 de outubro de 2014 \\ Florianópolis/SC}

Para determinar a energia de ativação da reação através da Equação 1 foram realizadas cinéticas destrutivas no intervalo de temperatura de 40 a $60{ }^{\circ} \mathrm{C}$. O sistema reacional para síntese de acetato de eugenila via catálise heterogênea foi composto de óleo essencial de folha de cravoda-índia (como fonte de eugenol) e anidrido acético, adicionados na razão molar de 1:3 (eugenol:anidrido acético), após dissolução completa dos substratos, o catalisador sólido foi adicionado na proporção de $12,5 \%$ ( $\mathrm{m} / \mathrm{m}$ de substratos) e o tempo reacional foi contado a partir da adição do mesmo, nas temperaturas estudadas. Após o término da reação, o catalisador foi filtrado em papel filtro e lavado com solvente ( $n$-hexano). Após evaporação do solvente em rotaevaporador, as amostras foram mantidas sob refrigeração $\left(4{ }^{\circ} \mathrm{C}\right)$ para posteriores análises cromatográficas.

\subsection{Determinação da conversão da reação}

A quantificação do éster produzido (acetato de eugenila) foi realizada por cromatografia gasosa (Shimadzu GC-2010). As análises foram realizadas utilizando coluna capilar de sílica fundida INOWAX (30 m x $250 \mu \mathrm{m}$ i.d.), 0,25 $\mathrm{m}$ de espessura de filme, detector FID, com a seguinte programação de temperatura: $40-180^{\circ} \mathrm{C}\left(3{ }^{\circ} \mathrm{C} \min ^{-1}\right), 180-230{ }^{\circ} \mathrm{C}\left(20{ }^{\circ} \mathrm{C} \mathrm{min}{ }^{-1}\right), 230{ }^{\circ} \mathrm{C}$ (20 min), temperatura do injetor $250{ }^{\circ} \mathrm{C}$, detector $275^{\circ} \mathrm{C}$, modo de injeção split, razão de split 1:100, gás de arraste $\mathrm{H}_{2}$, volume injetado $0,4 \mu \mathrm{L}$ de amostra diluída em diclorometano (1:10). A determinação da conversão das reações foi realizada acompanhando a redução na área do sinal do reagente limitante (eugenol).

\section{RESULTADOS E DISCUSSÃO}

A energia de ativação é a energia cinética mínima que os reagentes devem ter para que ocorra a formação de produtos. Portanto, processos com baixa energia de ativação ocorrem rapidamente, enquanto processos com elevada energia de ativação ocorrem mais lentamente. Para calcular a energia de ativação da acetilação do óleo essencial de cravo-da-índia com anidrido acético empregando peneira molecular $4 \AA$ como catalisador, utilizou-se a Equação de Arrhenius, sendo que para calcular as constantes de velocidade da Equação de Arrhenius foram realizadas 4 cinéticas destrutivas com temperaturas variando entre 40 e $60^{\circ} \mathrm{C}$, conforme mostra a Figura 1.

Através da Figura 1 pode-se observar que a conversão em ésteres aumenta com o aumento da temperatura e atinge conversão máxima de 96,6, 97,4 e 98,2\% em 360 minutos de reação para as temperaturas de 50,55 e $60{ }^{\circ} \mathrm{C}$, respectivamente. Temperaturas mais elevadas podem promover o aumento no número de colisões entre catalisador e substrato, resultando em uma reação mais acelerada.

A constante de velocidade da Equação de Arrhenius para as cinéticas apresentadas na Figura 1 foram calculadas a partir de linearização (ln concentração de substrato versus tempo) e estes valores foram empregados para calcular a energia de ativação (Ea) a partir da Equação de Arrhenius, conforme mostra a Figura 2. O uso da lei da velocidade de primeira ordem para calcular a constante de velocidade pode ser justificado pelo uso de um dos reagentes em excesso (anidrido acético), sendo que uma boa aproximação é considerar sua concentração constante. 
Desta forma, a velocidade da reação pode ser calculada a partir da concentração do reagente limitante (eugenol), o que caracteriza uma reação de primeira ordem.

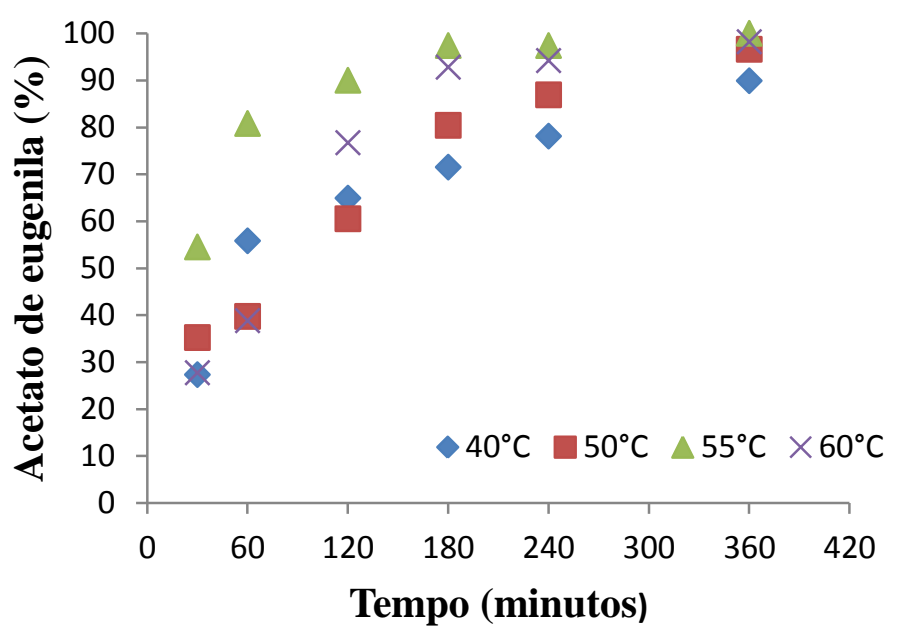

Figura 1 - Cinética da síntese de acetato de eugenila com peneira molecular variando a temperatura $\left(40,50,55\right.$ e $\left.60{ }^{\circ} \mathrm{C}\right)$, catalisador na proporção de $12 \%(\mathrm{~m} / \mathrm{m}$ de substratos $)$ e razão molar de eugenol e anidrido acético de 1:3.

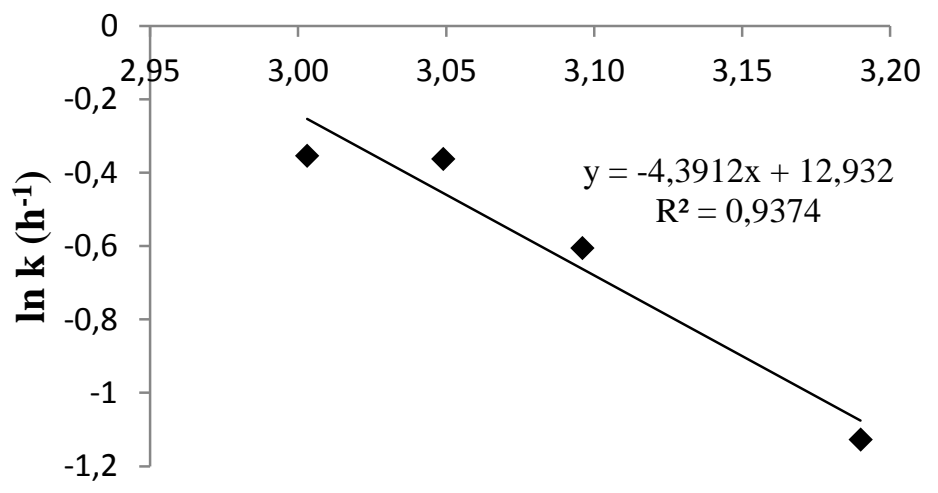

$$
\mathrm{T}^{-1}\left(10^{3} \mathrm{~K}^{-1}\right)
$$

Figura 2 - Arrhenius para a acetilação catalisada pela peneira molecular $4 \AA$ para a produção de acetato de eugenila.

A energia de ativação obtida na acetilação do óleo essencial de folha de cravo-da-índia com anidrido acético catalisada pela peneira molecular $4 \AA$ foi de $9,04 \mathrm{~kJ} \mathrm{~mol}^{-1}$. A energia de ativação obtida neste estudo foi menor que a encontrada por diversos autores. Basri et al. (2013), que obtiveram uma energia de ativação de $16,85 \mathrm{~kJ} \mathrm{~mol}^{-1}$, para a produção de etil ésteres a partir de 


\section{9 a 22 de outubro de 2014 \\ Florianópolis/SC}

óleo de palma, empregando Lipozyme TL IM. Yadav e Yadav (2012) avaliaram a produção de benzoato de eugenila por esterificação de eugenol com ácido benzóico catalisada por UDCaT-5, um catalisador sólido a base de dióxido de zircônio, a Ea para a reação foi de 39,22 $\mathrm{kJ} \mathrm{mol}^{-1}$. Akyalçın e Altıokka (2012), ao estudar a cinética da esterificação de ácido acético com 1-octanol catalisada por Amberlyst 36, obtiveram a energia de ativação da reação de $24,94 \mathrm{~kJ} \mathrm{~mol}^{-1}$. Khor et al.(2010) utilizaram a Lipozyme TL IM como catalisador na produção de étil ésteres a partir de óleo de palma e obtiveram energia de ativação de 22,16 kJ mol ${ }^{-1}$. Já Yu e Li (2005) obtiveram uma energia de ativação de $23,30 \mathrm{~kJ} \mathrm{~mol}^{-1}$ na esterificação de ácido propil galato e propanol, catalisada pela tanase de Aspergillus niger. Tendo em vista que um catalisador deve diminuir a energia necessária para que ocorra a reação, portanto, quanto menor o valor de energia de ativação, maior a velocidade de uma reação (Fennema, 1996), pode-se afirmar que peneira molecular $4 \AA$, mostrou-se um interessante catalisador para produção de acetato de eugenila. Porém, são necessários estudos adicionais, avaliando se em temperaturas mais elevadas ocorre ou não limitações de transferência de massa na reação, uma vez que esta limitação pode influenciar na obtenção de baixos valores de energia de ativação.

\section{CONCLUSÃO}

O aumento da temperatura da reação contribui para a obtenção de conversões mais elevadas, sendo que a temperatura de $50{ }^{\circ} \mathrm{C}$ favoreceu a obtenção de elevadas conversões em ésteres. A peneira molecular $4 \AA$ mostrou ser um catalisadsor interessante para síntese de acetato de eugenila, apresentando uma baixa energia de ativação.

\section{REFERÊNCIAS}

AKYALÇIN, S.; ALTIOKKA, M.R. Kinetics of esterification of acetic acid with 1-octanol in the presence of Amberlyst 36 Appl.Catal. A: Gen., v. 429-430, p. 70-84, 2012.

BASRI, M.; KASSIMA, M.A.; MOHAMADC, R.; ARIFF, A.B. Optimization and kinetic study on the synthesis of palm oil ester using Lipozyme TL IM. J.Mol. Catal. B: Enzym., v. 8586, p. 214-219, 2013.

BECK, J. S. Method for Synthesizing Mesoporous Crystalline Material, US Pat. 5057296, 1991.

COSTA, A .F. Farmacognosia, 5a ed., Lisboa: Fundação Caloust Gulbenkian, 1994.

FENNEMA, O.R. Food Chemistry. $3^{\mathrm{a}}$ ed. USA: Marcel Dekker, 1996.

DUMESIC, J.A.; HUBER, G.W.; BOUDART, M. Handbook of heterogeneous catalysis. $2^{\mathrm{a}}$ ed., Editado por: ERTL, G.; KNÖZINGER, H.; SCHÜTH, F.; WEITKAMP, J., Germany: Wiley-VCH, v. 1, 2008.

KHOR, G.K.; SIM J.H.; KAMARUDDIN A.H.; UZIR M.H. Thermodynamics and inhibition studies of lipozyme TL IM in biodiesel production via enzymatic transesterification Bioresour. Technol. v.101, p.6558-6561, 2010. 
KOLLMANNSBERGER, H.; NITZ, S. The flavour-composition of supercritical gas extracts: 2. Allpice (Pimenta dioica). Chemie Mikrobiologie Technologie der Lebensmittel; p.116-126, 1993.

KRESGE, C. T.;LEONOWICZ, M. E.; MICHAEL, E.; ROTH, W. J.; VARTULI, J.C. Synthetic Mesoporous Crystalline Material. US Pat. 5098684, 1992.

SARTORI, G.; BALLINI, R.; BIGI, F.; BOSICA, G.; MAGGI, R.; RIGHI, P. Protection (and deprotection) of functional groups in organic synthesis by heterogeneous catalysis. Chem. Rev., v. 104, p. 199-250, 2004.

YADAV, G.D.; YADAV, A.R. Insight into esterification of eugenol to eugenol benzoate using a solid super acidic modified zirconia catalyst UDCaT-5. Chem. Eng. J., v. 192, p. 146155,2012 .

YU, X.-W.; LI, Y.-Q. Lipase catalyzed transesterification of methyl acetoacetate with n-butanol J. Mol. Catal. B: Enzym., v.32, p.107-113, 2005.

WENDER, P. A.; HANDY, S. T.; WRIGHT, D. L. Chem. Ind. (London) 1997, 765. 